\title{
Active Noise Control of a Diesel Generator in a Luxury Yacht
}

\author{
Jordan Cheer*, Stephen J. Elliott \\ Institute of Sound and Vibration Research, University of Southampton, Southampton, \\ SO17 2LG, U.K.
}

\begin{abstract}
Active noise control has been applied to a variety of systems in order to improve performance without the increases in size and weight that would otherwise be required by traditional passive noise control treatments. This paper investigates the application of an active noise control system to the control of generator noise in the master cabin of a luxury yacht. A multichannel, multi-tonal active noise control system employing loudspeakers and microphones in the master cabin of the yacht is investigated. It is shown that, due to the high number of engine orders produced by the generator, in order to achieve significantly perceptible levels of noise attenuation it is necessary to control at least 7 individual orders. A controller is investigated which targets 19 engine orders and it is shown to achieve in excess of $5 \mathrm{~dB}$ broadband attenuation, whilst achieving up to $23 \mathrm{~dB}$ attenuation in individual orders. This corresponds to a $23 \%$ reduction in the Zwicker loudness.
\end{abstract}

Keywords: Active noise control, Zwicker loudness, Maritime environment

\section{Introduction}

Passive noise control treatments are an effective means of reducing the levels of noise and vibration experienced by humans in a variety of applications [1]. However, due to both weight and size restrictions their performance in practice is generally limited to the control of higher frequency noise and vibration. To overcome this limitation and achieve significant levels of low

\footnotetext{
*Corresponding author.

Email address: j.cheer@soton.ac.uk (Jordan Cheer)
} 
frequency noise attenuation, active control methods have been widely investigated $[2,3,4,5]$. Active control systems reduce the unwanted primary disturbance by the introduction of secondary sources, which produce either additional noise or vibration to control the original source.

Active control has been successfully demonstrated in a variety of engineering applications where the perception of the acoustic environment is particularly important. For example, in the aircraft environment the low frequency tonal noise induced by the propellers has been successfully controlled using a feedforward active noise control system, and a broadband attenuation in the sound pressure level of $7 \mathrm{dBA}$ has been reported [6]. In the automotive environment a variety of active control systems have been proposed for both engine $[7,8]$ and road noise control $[9,10]$. More broadly, active control technology has been applied to fan noise [11], active earmuffs [12], noise transmission through windows [13] and sound radiation from a helicopter transmission [14].

This paper investigates the application of active noise control to the attenuation of the noise produced by the generator in a luxury yacht, with the specific aim of creating a quiet region in the master cabin around the sleeping area. The application of active control technologies to the maritime environment has previously been investigated for vibration isolation [15, 16] and the control of radiating structural resonances [15]. More specifically, active noise control around the sleeping area in the cabin of a luxury yacht has been considered by Peretti et al [17]. In this previous work an adaptive feedback control system has been used to control the noise in the master cabin under a variety of operating conditions, including whilst stationary with the generator running and cruising at a variety of speeds. The control system used 4 error microphones, located at the headboard of the master cabin bed, and two sub-woofer loudspeakers, located under the bed. When the primary disturbance was produced by the generator alone, the sound field was dominated by a single tone at $25 \mathrm{~Hz}$ and the control system achieved $15 \mathrm{~dB}$ attenuation in this tone at a single error sensor. When the system was tested under cruising conditions, the primary disturbance generally contained a second tonal component related to the engine speed, and the adaptive feedback controller was able to achieve a similar level of narrowband attenuation in this case.

For tonal noise control problems, such as those due to engine and generator noise, a reference signal can often be obtained directly from a tachometer. Therefore, a feedforward control architecture can readily be employed. This 
paper investigates the use of a feedforward system to control the sound produced in the master cabin of a luxury yacht by the generator. In Section 2 the noise control problem is outlined. In Section 3 the feedforward active noise control system is described and in Section 4 the performance of this system is presented in terms of both the attenuation and the reduction in perceived loudness. Finally, conclusions are drawn in Section 5.

\section{Diesel Generator Noise Control Problem}

The acoustic environment in high quality luxury yachts has become an important consideration for yacht manufacturers and may be seen as a potentially distinguishing feature between two different high quality products. High levels of noise and vibration can result in an uncomfortable environment and, therefore, there is a desire to achieve low levels of noise and vibration throughout the living quarters of luxury yachts. At mid to high frequencies passive noise and vibration control treatments can be employed to achieve a quiet living space on-board. At low frequencies, however, the size and weight of passive treatments becomes too large and is often either impractical or significantly increases the weight of the yacht, which in turn limits its speed and efficiency. Although occupants may accept the levels of noise and vibration produced by the yacht's engines whilst underway, they may be more sensitive to the noise produced by the electrical generator whilst moored. This is due to the low levels of background noise in this scenario and this is a particular problem when the occupants are trying to sleep. Therefore, focus has been made on controlling the noise produced by the generator in the master cabin.

Figure 1 shows the layout of the real yacht considered in this work. The yacht is a $60 \mathrm{ft}$ vessel and the location of the generator in the engine room, which is adjacent to the master cabin, is shown in Figure 1. In order to characterise the noise produced by the generator, the sound pressure level has been measured at the head of the bed in the master cabin using the microphones shown in Figure 1 when the diesel generator is running. Figure 2 shows the spectrum of the A-weighted sound pressure level measured at microphone number 2. The pressure has been plotted in decibels relative to an arbitrary constant due to commercial sensitivity, however, it should be highlighted that the sound pressure level is relatively low, but is disturbing when the background environmental noise level is low. From the results shown in Figure 2 it can be seen that the noise spectrum is characterised 
by a large number of tonal components, which comprise a full series of both integer and non-integer harmonics of the fundamental engine order at the mains frequency of $50 \mathrm{~Hz}$. This tonal disturbance has been reported to be subjectively disturbing and, therefore, in the following sections the feasibility of using active control to reduce the overall sound pressure level will be explored.

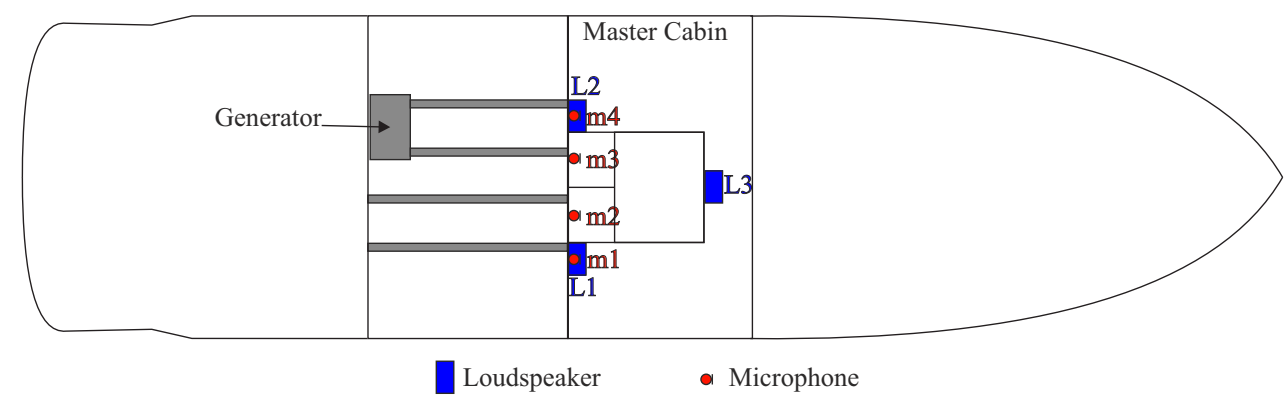

Figure 1: The layout of the standby diesel generator and the control system components on the yacht.

\section{Feedforward Active Noise Control System}

The active control of tonal noise has been investigated for a number of different applications [6, 7], as discussed in the introduction. An active tonal noise control system can be efficiently implemented using a feedforward controller and the most commonly employed algorithm is the filtered reference, or filtered- $x$, Least Mean Squares (LMS) algorithm [5]. The multichannel formulation of this algorithm was originally presented in the late 1980s [18] and has since been used in a variety of applications. The block diagram in Figure 3 shows the general outline of the multichannel feedforward control system. In this block diagram e is the vector of error signals, which the controller attempts to minimise, $\mathbf{d}$ is the vector of disturbance signals, $\mathbf{u}$ is the vector of control signals, $x$ is the reference signal, $\mathbf{R}$ is the matrix of filtered reference signals, $\mathbf{G}$ represents the physical responses between the control sources and the error sensors, $\widehat{\mathbf{G}}$ is a model of this plant response used in the control update algorithm, $\mathbf{W}$ represents the control filters and $\alpha$ is the convergence gain which determines the speed of adaptation for the controller. 


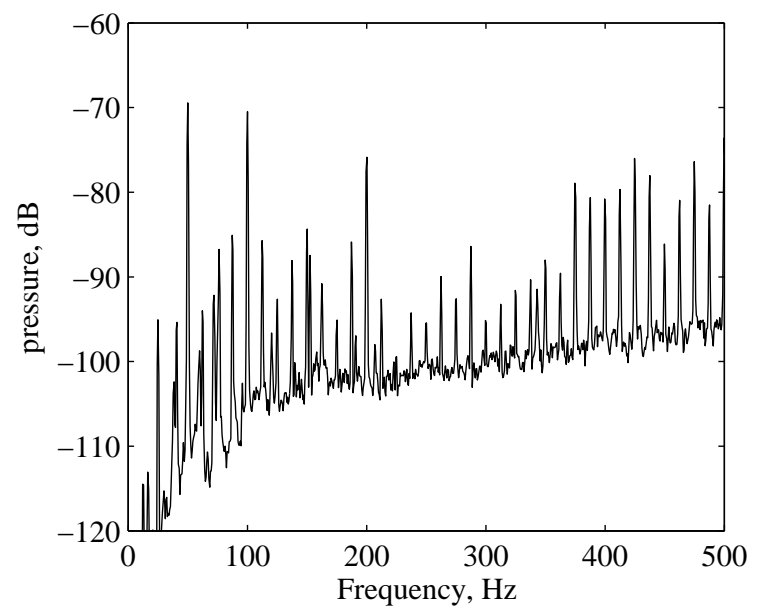

Figure 2: The A-weighted sound pressure level measured in the master cabin at the headboard of the bed when the diesel generator is running plotted in decibels relative to an arbitrary reference.

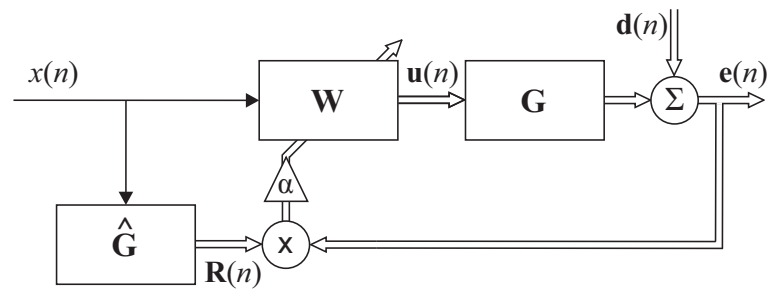

Figure 3: The filtered- $x$ LMS feedforward active noise control algorithm blockdiagram. 
For a multichannel control system with $L$ error sensors, $M$ secondary sources and control filters with $I$ coefficients, the control filter coefficients can be adapted to minimise the sum of the squared error signals according to the leaky filtered- $x$ LMS algorithm, which is

$$
\mathbf{w}(n+1)=\beta \mathbf{w}(n)-\alpha \mathbf{R}^{T}(n) \mathbf{e}(n),
$$

where $\mathbf{w}$ is the vector of $M I$ control filter coefficients and $\beta$ is the leakage coefficient. The inclusion of the leakage coefficient, $\beta$, improves the robustness of the algorithm and, therefore, is necessary in practice to ensure long term performance. A full description and analysis of this algorithm is widely available in the literature $[5,19]$ and, therefore, will not be reproduced here. However, it is important to highlight that the single frequency, tonal controller can be efficiently implemented using two-coefficient control filters [5, 19] and that control of multiple tones can be simply achieved by employing multiple single tonal controllers operating in parallel, as shown in Figure 4. In this case, each channel of the plant model corresponding to each tone, $\hat{\mathbf{G}}_{k}$, can also be implemented using a two-coefficient filter. This is possible assuming that the disturbance frequency is constant and, therefore, an accurate plant model is only required at the control frequency. If there is some variation in the control frequencies, it may be possible to achieve an increase in performance by using more than two-coefficients in the control and plant modelling filters. However, this comes at the cost of increased computational demand, and in the generator control application considered here, a larger increase in the overall performance was achievable by using the available computational power to increase the number of orders controlled using two-coefficient filters.

For the generator active noise control application in the yacht, the control system consists of 4 error microphones located on the headboard of the bed in the master cabin and three control loudspeakers, positioned as shown in Figure 1. In order to implement the filtered- $x$ LMS algorithm it is necessary to have a model of the plant responses between the control loudspeakers and the error microphones, which is shown as $\widehat{\mathbf{G}}$ in Figure 3. Therefore, these responses have been measured in the yacht by driving each of the loudspeakers with broadband pink noise. The resulting frequency responses between each of the loudspeakers and error microphone number 1 are shown as an example in Figure 5 and the impulse response between loudspeaker 1 and microphone 1 is shown for example in Figure 6 . From these results it can be seen that the acoustic environment is relatively well damped with 


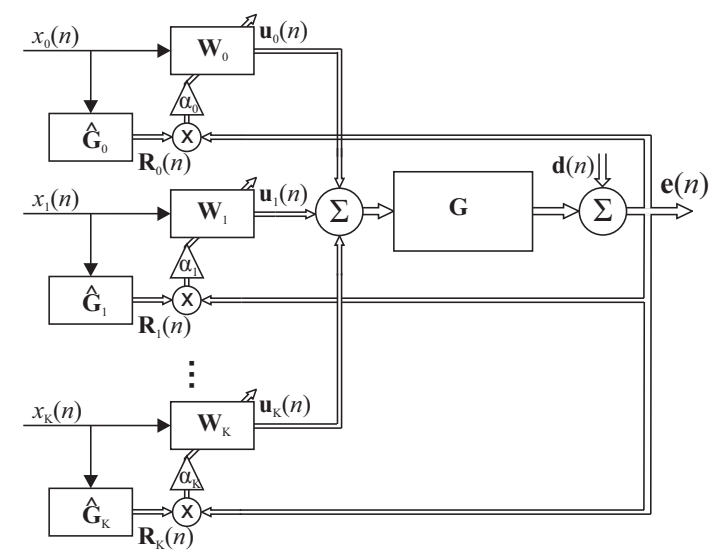

Figure 4: The multitonal feedforward active noise control algorithm implemented using $K$ parallel single frequency controllers.

a reverberation time of around $180 \mathrm{~ms}$ and, due to the size of the cabin, is characterised by a large number of modes.

In a modal acoustic environment, such as the yacht cabin, global active noise control is limited by how well the secondary sources couple into the modes of the enclosure [2]. A rough indication is that, in order to achieve global control of the sound field in the enclosure, the number of secondary sources must be around equal to the number of acoustic modes excited. Therefore, in a modally dense acoustic environment, global control quickly becomes impractical as the number of required secondary sources increases approximately in proportion to the cube of the excitation frequency [5]. As a result of this physical limitation, active control in large enclosures is often limited to controlling the sound field in a specific local region and a significant amount of research has been conducted in this area [2, 20, 21, 22, 23]. For a local active control system employing a single error sensor and remote secondary source and operating in a diffuse sound field, it has been shown that the diameter of the zone of quiet within which $10 \mathrm{~dB}$ of attenuation is achieved is limited to about one tenth of the acoustic wavelength [22]. Therefore, at $250 \mathrm{~Hz}$ the zone of quiet would have a diameter of around $14 \mathrm{~cm}$. However, it has also been shown that, by employing multiple error sensors and secondary sources, the size of the zone of quiet can be significantly increased [23]. The specific size and shape of the zone of quiet in practice, however, will depend on the primary sound field, the acoustic environment 

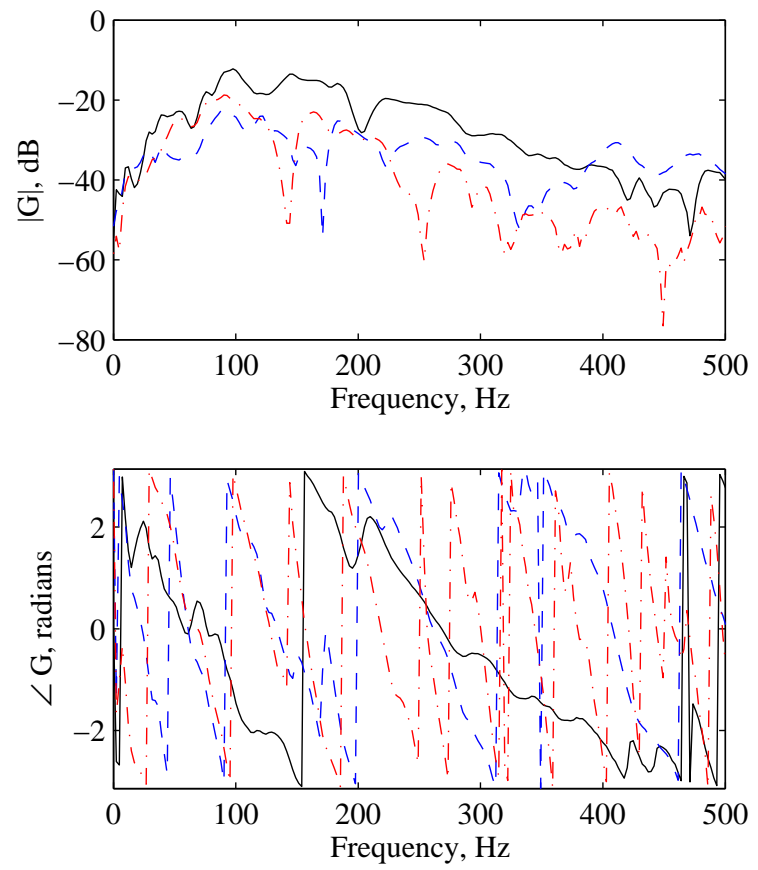

Figure 5: Plant responses between each of the three control loudspeakers and microphone 1. 


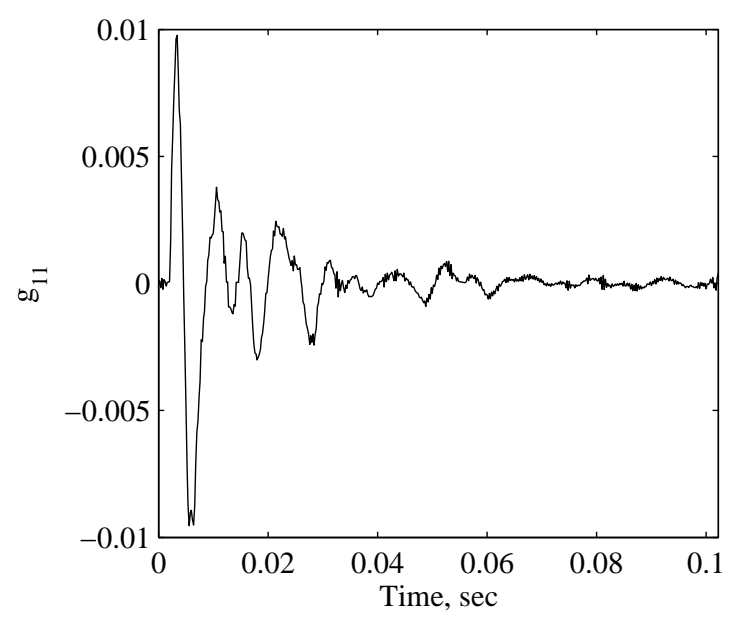

Figure 6: Impulse response between loudspeaker 1 and microphone 1.

and the geometry of the error sensors and secondary sources. Nevertheless, it is unlikely that a zone of quiet with dimensions large enough to allow significant movement of those benefiting from the noise attenuation will be achievable much beyond around $250 \mathrm{~Hz}$. In the present application, by locating the error sensors at the head of the bed, the focus of the control system is to reduce the sound pressure level around the head of the occupant of the bed and control will only be attempted up to $250 \mathrm{~Hz}$.

From the pressure spectrum shown in Figure 2, it is clear that to achieve a perceptually significant level of noise attenuation it will be necessary to control multiple tones. As discussed above, and shown in Figure 4, this can be achieved using multiple single frequency filtered- $x$ LMS controllers operating in parallel. However, what is perhaps not straightforward is how to set the convergence gain, $\alpha$, for each of the multichannel single frequency controllers, without a significant degree of trial and error. This parameter is particularly important as it governs the trade-off between speed of convergence and the stability of the controller. If the convergence gain is too high the controller will be unstable, whereas, if the convergence gain is too low the controller will take a long time to converge and achieve a significant level of attenuation.

For a single frequency controller operating at the angular frequency $\omega_{c}$, it has been shown that the maximum magnitude of the convergence gain must 
be less than [5]

$$
\alpha_{\max }\left(\omega_{c}\right)=\frac{2}{\lambda_{\max }\left(\omega_{c}\right)}
$$

where $\lambda_{\max }\left(\omega_{c}\right)$ is the maximum eigenvalue of $\mathbf{G}^{H} \mathbf{G}$ and $\mathbf{G}$ is the matrix of plant responses at the frequency of control. This condition for the convergence of the adaptive algorithm is based on the assumption that the error signals have reached their steady-state values at each iteration and, therefore, generally over estimates the maximum convergence gain. However, practical experience has shown that the convergence gains for the multiple single frequency controllers can be calculated according to eq. 2 and then scaled using a single scaling parameter as

$$
\alpha\left(\omega_{c}\right)=\gamma \alpha_{\max }\left(\omega_{c}\right)
$$

where $\gamma$ is generally a small positive constant that is set manually by the system designer to provide a trade-off between convergence speed and stability for all of the single frequency controllers in parallel. A small value of $\gamma$ ensures that the controller is stable, but it will be slow to converge and may not reach the optimal solution in practical applications. By increasing $\gamma$ a faster convergence speed can be achieved, but the controller will be less robust to practical uncertainties and will become unstable when $\gamma$ is too large. In the yacht application considered here $\gamma$ has been set at $1 \times 10^{-3}$, which ensures the convergence of the error signals does not become oscillatory.

For the plant responses measured in the yacht, Figure 7 shows the variation in $\alpha_{\max }$ against frequency. From this plot it can be seen that there are significant variations in $\alpha_{\max }$ against frequency and this highlights the benefit of calculating the convergence gain for each single frequency controller according to eq. 2 and then scaling all of the gains according to eq. 3 rather having to set the convergence gain for each single frequency controller manually.

\section{Performance Assessment}

Due to the large number of harmonics present in the noise spectrum produced by the generator, as shown in Figure 2, it is expected that in order to achieve perceptually significant levels of noise attenuation, multiple tones will need to be controlled. To assess this requirement, simulations using the multichannel plant response and the disturbance signals measured in the 


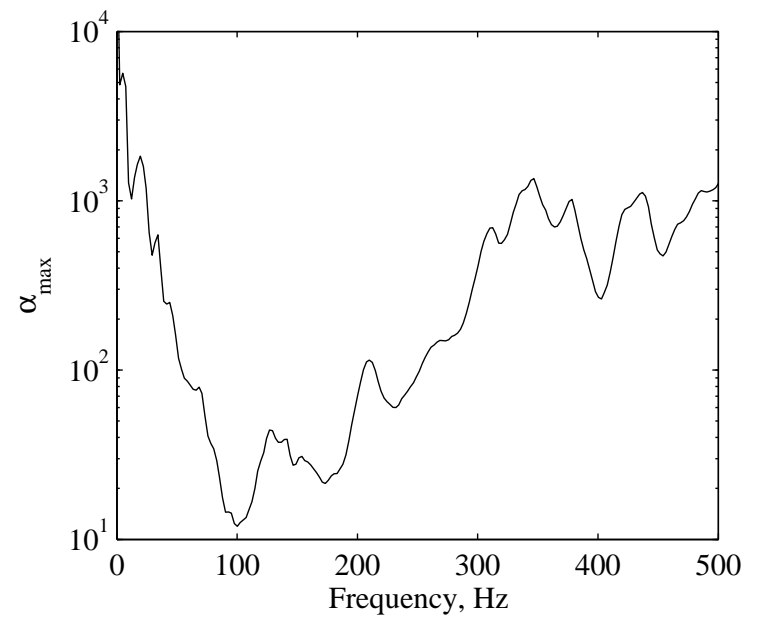

Figure 7: The theoretical maximum convergence gain, $\alpha_{\max }$, over frequency.

yacht have initially been conducted. Figure 8 shows the broadband average attenuation in the A-weighted sound pressure level as the number of orders, or harmonics controlled is increased from 1 to 19. The engine orders are controlled in order of decreasing level - i.e. the highest level order is controlled first and then the two highest level orders are controlled and so forth. The first order which is controlled is at $50 \mathrm{~Hz}$ and more than $23 \mathrm{~dB}$ attenuation is achieved at this single order, however, from the results in Figure 8 it can be seen that the broadband attenuation is only $1.8 \mathrm{~dB}$. As the number of orders controlled is increased, the level of broadband attenuation also increases and, from Figure 8, it can be seen that the level of broadband attenuation reaches $5 \mathrm{~dB}$ when 7 orders are controlled. This change in level is generally considered to be clearly perceptible, although the specific details of the perceived change in level will also be dependent on the specific characteristics of the spectral and temporal content [24]. As the number of orders controlled is increased from 7 up to the maximum considered of 19, the increase in broadband performance reduces and a maximum broadband performance of $5.7 \mathrm{~dB}$ is achieved. It is also worth highlighting that the performance of the controller at the individual orders is not significantly affected when the number of orders is increased; that is, $23 \mathrm{~dB}$ attenuation is achieved at $50 \mathrm{~Hz}$ when this order is controlled in isolation and when all 19 orders are controlled.

Figure 9 shows the spectrum of the sum of the squared error signals mea- 


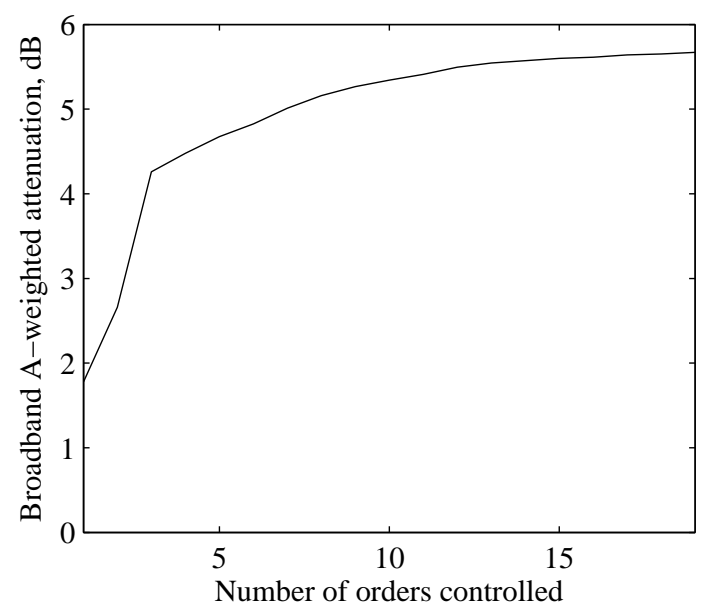

Figure 8: The broadband attenuation in the cost function as the number of controlled engine orders is increased.

sured at the four error microphones after convergence when the 19 orders are controlled. From this plot it can be seen that high levels of narrowband attenuation are achieved at certain orders, for example, more than $20 \mathrm{~dB}$ attenuation is achieved at the high level orders at 50 and $100 \mathrm{~Hz}$. However, the attenuation in other orders is rather limited, for example, the attenuation at $250 \mathrm{~Hz}$ is only around $4 \mathrm{~dB}$. That said, it can be seen from Figure 9 that, in general, the feedforward controller achieves significant levels of attenuation in the A-weighted sound pressure level. It is important to highlight that the results in Figure 9 correspond to the reductions at the error microphone locations, however, as discussed in the previous section, as the control frequency increases the size of the zone of quiet will become smaller. Therefore, the pressure would ideally be measured throughout the region where a person's head might be located in order to confirm that sufficient control was achieved at all positions. This was not feasible during the onboard measurements, however, based on the previous work on local active control with multichannel systems discussed in the previous section [23], it is expected that at the maximum control frequency of $250 \mathrm{~Hz}$ the zone of quiet will extend over the region where the person's head is likely to be whilst lying on the bed.

In addition to the steady-state performance shown in Figure 9, it is also 


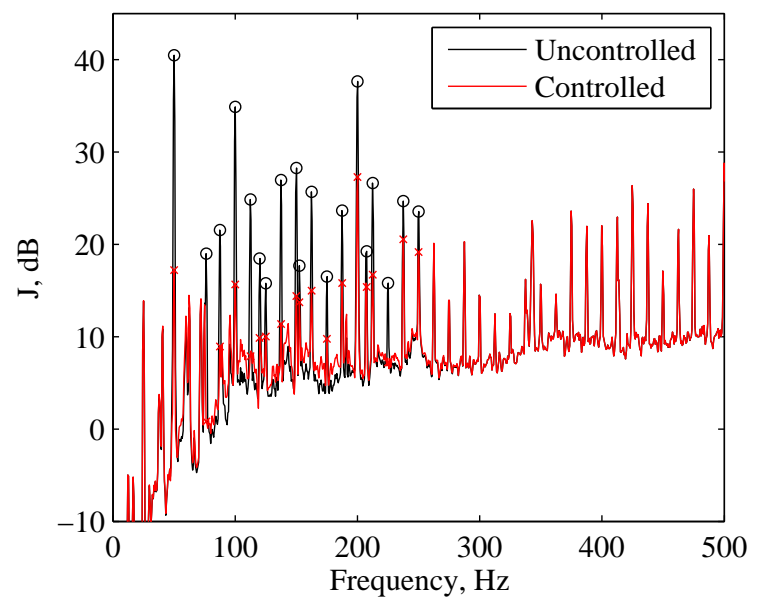

Figure 9: The sum of the squared error sensor signals, $J$, before an after control. The open circles show the level of the targeted order before control and the crosses show the level of the targeted orders after control.

interesting to observe the convergence behaviour of the controller. Therefore, Figure 10 shows the convergence of the sum of the squared error signals at the five highest level orders. From this plot it can be seen that the orders corresponding to 100, 137.5, 150 and $200 \mathrm{~Hz}$ all converge to their final levels of attenuation in under 2 seconds. Slightly different behaviour can be seen for the order at $50 \mathrm{~Hz}$, which shows two clear convergence slopes, which can be related to the eigenvalue spread of the plant matrix [5]. The first $10 \mathrm{~dB}$ of control is achieved in under 2 seconds, whilst it takes a further 8 seconds to achieve the full $23 \mathrm{~dB}$ of attenuation. The speed of convergence is suitable for the generator noise control problem, since the speed of the generator does not vary rapidly, unlike in the application of active noise control to road vehicle engine noise attenuation where extremely fast convergence speeds are required.

In order to assess whether the levels of attenuation in the A-weighted sound pressure level will be perceptually significant, the Zwicker loudness model [24] has been used to calculate the percentage reduction in loudness as the number of engine orders controlled is increased. The Zwicker loudness model involves filtering the error signals to account for the transmission through the outer and middle ear, calculation of the basilar membrane excita- 


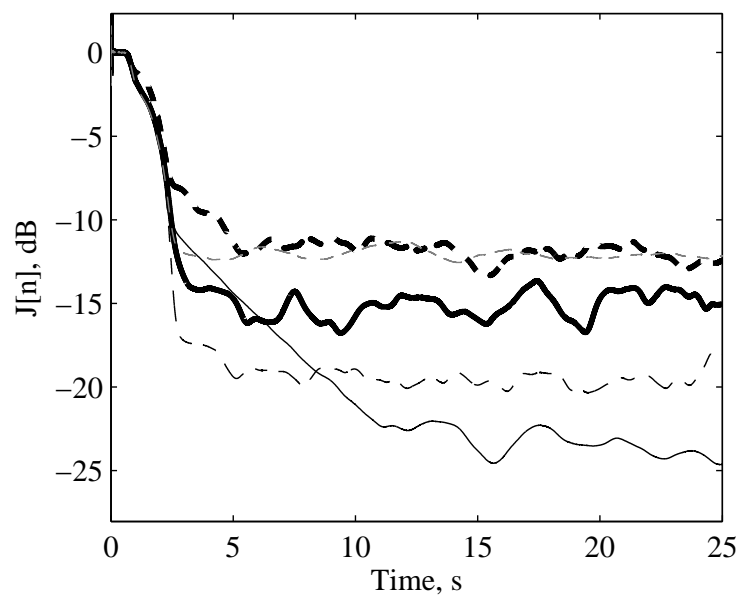

Figure 10: The convergence of the sum of the squared error sensor signals, $J$, at the five dominant orders. $50 \mathrm{~Hz}$ (thin solid line); $100 \mathrm{~Hz}$ (thin dashed line); $137.5 \mathrm{~Hz}$ (thick solid line); $150 \mathrm{~Hz}$ (thick dashed line); $200 \mathrm{~Hz}$ (thin light dashed line).

tion pattern, transformation of the excitation pattern to the specific loudness and finally, integration of the specific loudness over Bark gives the Loudness in Sones. This loudness model has previously been used to assess the performance of active control systems aimed at controlling automotive engine noise $[8,25]$. It is important to use such a psychoacoustic model when assessing the performance of active control systems, since the often employed A-weighted sound pressure level generally underestimates the loudness due to low frequency components [26], such as those present in most active control applications including the generator noise considered here. Figure 11 shows the percentage reduction in the calculated loudness as the number of engine orders controlled is increased. From this plot it can be seen that a $10 \%$ reduction in the loudness is achieved by controlling a single engine order, while controlling 19 orders results in a $23 \%$ loudness reduction. From these results it can be seen that, for the noise produced by the generator, the reductions in broadband A-weighted sound pressure level achieved by the feedforward active control system do translate into significant reductions in the perceived loudness. 


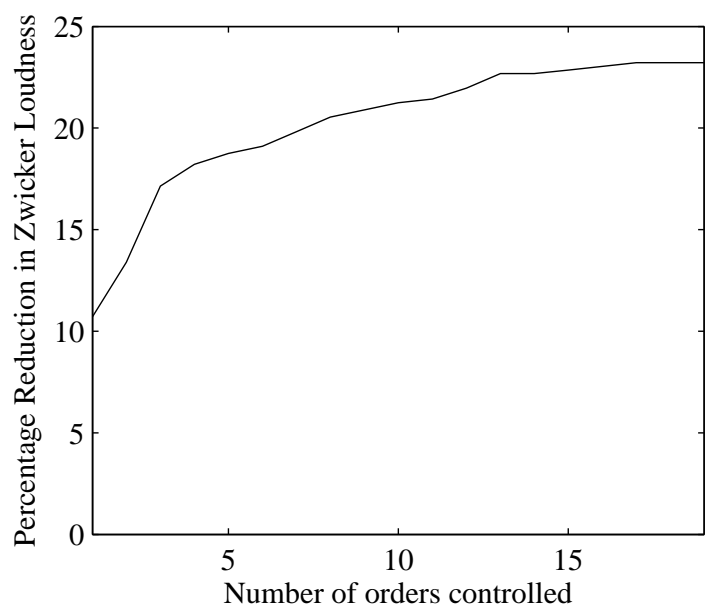

Figure 11: The percentage reduction in the Zwicker loudness as the number of controlled engine orders is increased.

\section{Conclusions}

In this paper the potential of applying an active noise control system to reduce the levels of noise produced by a diesel generator in the master cabin of a luxury yacht has been investigated. The noise control problem has first been investigated and it has been shown that the noise and vibration due to the generator produce a sound pressure spectrum in the master cabin containing a full series of both integer and non-integer engine orders. It is difficult to control this further using passive control treatments due to both weight and size limitations. Global active noise control is also not feasible due to the relatively wideband frequency content and the modally dense nature of the master cabin. Therefore, a practical active control system has been implemented which focuses a zone of control at the head of the bed, where the generator noise is most disturbing when occupants are trying to sleep.

It has been shown that, due to the large number of tonal components present in the disturbance noise spectrum, it is necessary to control multiple tones in order to achieve a significant level of broadband attenuation. Therefore, a feedforward control system has been investigated which controls up to 19 engine orders. A straightforward method of defining the convergence gain for each of the single tone controllers has been presented, which requires the tuning of a single parameter rather than 19. It has been shown that this 
controller achieves significant broadband attenuation in the sound pressure level at the error microphones located at the head of the bed and through the use of the Zwicker loudness model it has also been shown that this translates into a $23 \%$ reduction in the loudness.

\section{Acknowledgement}

The authors gratefully acknowledge the assistance of Princess Yachts in carrying out the measurements used in this paper. This work was supported by the Innovate UK project 101004 .

\section{References}

[1] D. A. Bies, C. H. Hansen, Engineering noise control: theory and practice, 4th Edition, Spon Press, Oxford, U.K., 2009.

[2] P. Nelson, S. J. Elliott, Active Control of Sound, Academic Press, London, 1992.

[3] C. R. Fuller, S. J. Elliott, P. A. Nelson, Active Control of Vibration, Academic Press, London, 1996.

[4] S. M. Kuo, D. R. Morgan, Active noise control: A tutorial review, Proceedings of the IEEE 87 (6) (1999) 943-973.

[5] S. J. Elliott, Signal Processing for Active Control, Academic Press, London, 2001.

[6] S. Elliott, P. Nelson, I. Stothers, C. Boucher, In-flight experiments on the active control of propeller-induced cabin noise, Journal of Sound and Vibration 140 (2) (1990) 219 - 238.

[7] S. J. Elliott, I. M. Stothers, P. Nelson, M. A. McDonald, D. C. Quinn, T. J. Saunders, The active control of engine noise inside cars, in: M. Bockhoff (Ed.), Proceedings of INTER-NOISE 88, Vol. 2, Poughkeepsie, New York, 1988, pp. 987-990.

[8] L. P. R. de Oliveira, K. Janssens, P. Gajdatsy, H. Van der Auweraer, P. S. Varoto, P. Sas, W. Desmet, Active sound quality control of engine induced cavity noise, Mechanical systems and signal processing 23 (2009) 476-488. 
[9] T. J. Sutton, S. J. Elliott, M. A. McDonald, T. J. Saunders, Active control of road noise inside vehicles, Journal of Noise Control Engineering 42 (4) (1994) 137-146.

[10] J. Cheer, S. J. Elliott, Multichannel control systems for the attenuation of interior road noise in vehicles, Mechanical Systems and Signal Processing 60-61 (2015) $753-769$.

[11] K. Chen, R. Paurobally, J. Pan, X. Qiu, Improving active control of fan noise with automatic spectral reshaping for reference signal, Applied Acoustics 87 (2015) $142-152$.

[12] S.-P. Moon, W. L. Lee, Jeong, T.-G. Chang, Performance analysis of an adaptive feedback active noise control based earmuffs system, Applied Acoustics 96 (2015) 53-60.

[13] T. Pamies, J. Romeu, M. Genesca, R. Arcos, Active control of aircraft fly-over sound tranmission through an open window, Applied Acoustics 84 (2014) 116-121.

[14] P. Belanger, A. Berry, Y. Pasco, O. Robin, Y. St-Amant, S. Rajan, Multi-harmonic active structural acoustic control of a helicopter main transmission noise using the principal component analysis, Applied Acoustics 70 (1) (2009) $153-164$.

[15] S. Daley, F. Johnson, J. Pearson, R. Dixon, Active vibration control for marine applications, Control Engineering Practice 12 (4) (2004) 465 474 .

[16] M. Kauba, S. Herold, T. Koch, D. Mayer, T. Melz, Design and application of an active virbation control system for a marine engine mount, in: International Conference on Noise and Vibration Engineering, 2008, pp. 241-255.

[17] P. Peretti, S. Cecchi, L. Romoli, F. Piazza, Adaptive feedback active noise control for yacht environments, Control Systems Technology, IEEE Transactions on 22 (2) (2014) 737-744.

[18] S. J. Elliott, I. M. Stothers, P. A. Nelson, A multiple error LMS algorithm and its application to the active control of sound and vibration, 
IEEE Transactions on Acoustics, Speech, and Signal Processing 35 (10) (1987) 1423-1434.

[19] S. M. Kuo, D. Morgan, Active noise control systems: Algorithms and DSP Implementations, Wiley, 1996.

[20] M. De Diego, A. Gonzalez, Performance evaluation of multichannel adaptive algorithms for local active noise control, Journal of Sound and vibration 244 (4) (2001) 615-634.

[21] W.-K. Tseng, B. Rafaely, S. Elliott, Local active sound control using 2norm and-norm pressure minimization, Journal of Sound and Vibration 234 (3) (2000) 427-439.

[22] S. J. Elliott, P. Joseph, A. J. Bullmore, P. A. Nelson, Active cancellation at a point in a pure tone diffuse sound field, Journal of Sound and Vibration 120 (1988) 183-189.

[23] S. J. Elliott, J. Cheer, Modeling local active sound control with remote sensors in spatially random pressure fields, Journal of the Acoustical Society of America 137 (4) (2015) 1936-1946.

[24] H. Fastl, E. Zwicker, Psychoacoustics: Facts and models, Vol. 22, Springer Science \& Business Media, 2007.

[25] A. Gonzalez, M. Ferrer, M. De Diego, G. Pinero, J. Garcia-Bonito, Sound quality of low-frequency and car engine noises after active noise control, Journal of Sound and Vibration 265 (3) (2003) 663-679.

[26] H. Fastl, The psychoacoustics of sound quality evaluation, Acustica 83 (1997) 754-764. 